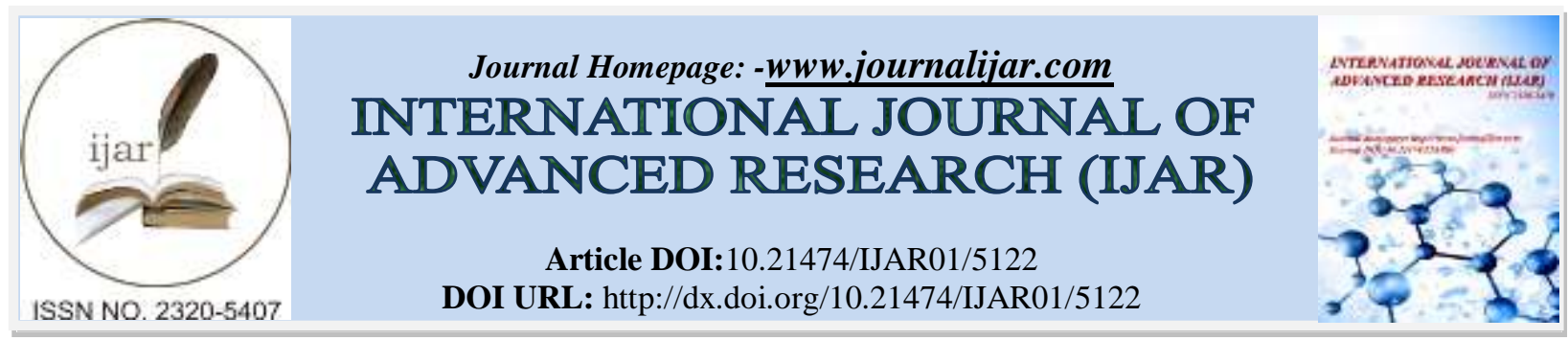

RESEARCH ARTICLE

\title{
A STUDY OF CAROTID INTIMA-MEDIA THICKNESS and RETINAL ARTERY CHANGES IN PATIENTS WITH NON ALCOHOLIC FATTY LIVER DISEASE.
}

\section{Dr. Prabhu. T. $\mathbf{M}^{\mathbf{1}}$ and Dr .S. Krishnasamy Prasad ${ }^{2}$.}

1. Senior resident in General Medicine ,Government Rajaji Hospital and Madurai Medical College, Madurai.

2. Asst Professor, Department of General Medicine, Government Rajaji Hospital and Madurai Medical College, Madurai.

\section{Manuscript Info}

Manuscript History

Received: 09 June 2017

Final Accepted: 11 July 2017

Published: August 2017

\section{Abstract}

Non-Alcoholic Fatty Liver Disease (NAFLD) is one of the most common liver diseases reported all over the world and the disease spectrum ranges from simplesteatosis to non-alcoholic steato-hepatitis to cirrhosis . NAFLD has strong association with metabolic syndrome. The carotid intima-media thickness (CIMT) is considered to be a noninvasive marker for atherosclerosis andthe thickness is directly proportional to the presence of atherosclerosis in the coronary vessels and the severity of cardiovascular disease.

Aims And Objectives:1. To measure the thickness of Carotid Intima Media (CIMT) in patients with Non-Alcoholic Fatty Liver Disease (NAFLD) and compare it with Controls, to establish the strong association of NAFLD with increased CIMT and to prove that NAFLD an independent risk factor for cardiovascular morbidity..

2. To look for any associated retinal vascular changes in NAFLD

Materials And Methods: This study will be conducted between June 2016 to November 2016, among Patients with non alcoholic fatty liver who are admitted in General Medicine Department Of Government Rajaji Hospital, Madurai and equal number of age matched controls.

Results: We found the strong association between NAFLD and carotid intima media thickness as $\mathrm{p}$ value is 0.001 . Mean carotid intima media thickness in NAFLD is $0.9284 \mathrm{~mm}$ compared to 0.6695 in control group. We also found the strong association between NAFLD and retinal vascular changes as $\mathrm{p}$ value is 0.004 .12 patients have retinal changes in NAFLD group compared to 2 patients in control group.

Conclusion: Non-Alcoholic Fatty Liver Disease (NAFLD) is one of the most common liver diseases reported all over the world.Carotid Intima Media Thickness (CIMT) is a useful tool for detection of subclinical atherosclerosis. In our study, we found that, there is strong association between, NAFLD and Carotid intima media thickness, Retinal artery changes.

So all patients with NAFLD should be investigated with carotid Doppler, and they all should treated with statins and other cardioprotective drugs to prevent cardiovascular complication. They all should get treatment for obesity and with insulin sensitizers to prevent the progression of NAFLD. This simple and non-invasive practice will 
help in early diagnosis ofcardiovascular disease, especially in overt cases.

Copy Right, IJAR, 2017,. All rights reserved.

\section{Introduction:-}

Carotid Intima Media Thickness (CIMT) is a useful tool for detection of sub-clinical atherosclerosis. It therefore, not only indirectly indicates the presence of coronary atherosclerosis but also gives some estimation of its severityNonAlcoholic Fatty Liver Disease (NAFLD) is one of the most common liver diseases reported all over the world and the disease spectrum ranges from simple steatosis to non-alcoholic steato-hepatitis to cirrhosis Fatty liver, the common term used for NAFLD has strong association with metabolic syndrome. Obesity, type-2 diabetes, dyslipidemia and insulin resistance is therefore evident in most of these patients . As there is a clear association of fatty liver disease being a part of spectrum of metabolic syndrome, several studies have been conducted to establish NAFLD as an independent risk factor for atherosclerosis and cardiovascular disease.

The CIMT is considered to be a non-invasive marker for atherosclerosis and the thickness is directly proportional to the presence of atherosclerosis in the coronary vessels and the severity of cardiovascular disease. The aim of our study is to determine the relationship between NAFLD and CIMT and retinal artery changes by comparing the CIMT of patients and fundus chages with sonographically proven NAFLD with a group of patients having normal echogenicity of liver parenchyma on ultrasound.

\section{Study Population:-}

This study will be conducted between June 2016 to November 2016, among Patients with non alcoholic fatty liver who are admitted in General Medicine Department Of Government Rajaji Hospital, Madurai and equal number of age matched controls.

\section{Inclusion criteria:-}

Sonographically proven fatty liver.,Age - adults > 30 , Gender - both male and femaleNon alcoholic or alcoholic with $<20 \mathrm{~g} /$ day consumption

\section{Exclusion criteria:-}

Patients who have problems for abdominal ultrasonography, Those who are using steroid in diseases such as bronchial asthma, rheumatoid arthritis and Intestinal Bowel Disease (IBD) andThose who are being treated with drugs affecting laboratory results, for example, aspirin, statins, fibrates and metformin,those who have a history of liver disorders such as HBV or HCV, infection, Those who are consuming more than $20 \mathrm{gm} / \mathrm{day}$ of alcohol were Excluded.

\section{Method:-}

Fifty patients with NAFLD confirmed on abdominal ultrasound and 50 controls with normal liver parenchyma satisfying the inclusion and exclusion criteria are evaluated.

The collected data were subjected to statistical analysis using Statistical package for Social sciences [SPSS version 19.0 (SPSS Inc., Chicago, IL, USA)]. Categorical data were presented as numbers and percentages, whereas continuous data were expressed as mean and standard deviation (SD) or median and inter quartile range.

The continuous data was assessed for normality by Shapiro-wilk test. The patternof distribution of each variable was found to be in non normal distribution thusnon-parametric Mann-Whitney $\mathbf{U}$ test was indicated as appropriate test. 


\section{Results and Interpretation:-} Age distribution among control

Age distribution among NAFLD

\begin{tabular}{|l|l|l|}
\hline AGE & NAFLD & PERCENTAGE \\
\hline $31-40$ & 11 & $22 \%$ \\
\hline $41-50$ & 17 & $34 \%$ \\
\hline $51-60$ & 17 & $34 \%$ \\
\hline-60 & 5 & $10 \%$ \\
\hline total & 50 & $100 \%$ \\
\hline
\end{tabular}

\begin{tabular}{|l|l|l|}
\hline AGE & CONTROL & PERCENTAGE \\
\hline $31-40$ & 12 & $24 \%$ \\
\hline $41-50$ & 20 & $40 \%$ \\
\hline $51-60$ & 17 & $34 \%$ \\
\hline$>60$ & 1 & $2 \%$ \\
\hline total & 50 & $100 \%$ \\
\hline
\end{tabular}

Gender DistribtuionInNafld Patients:-

In this , among NAFLD patients, $60 \%$ were females and $40 \%$ males

\section{Gender DistribtuionIn Control:-}

In this study, among controls $74 \%$ were males and $26 \%$ were females. In NAFLD patients, $38 \%$ were diabetes mellitus and $16 \%$ were diabetes among control .Among NAFLD patients 16\% were obese and among control 8\% were obese.

Hypertensive patients are equal in number both in NAFLD and control.

Distribution Of Retinal Artery Changes In Nafld And Control

\begin{tabular}{|l|l|l|}
\hline & Retinal artery changes & percentage \\
\hline NAFLD & 12 & $24 \%$ \\
\hline CONTROLS & 2 & $4 \%$ \\
\hline TOTAL & 14 & $28 \%$ \\
\hline
\end{tabular}

Among NAFLD patients, retinal artery changes are observed in $24 \%$ of the patients, Among control, retinal artery changes observed only in $4 \%$ of the patients

\section{Comparison Of Carotid Intima Media Thickness InNafld:-}

\begin{tabular}{|l|l|}
\hline MEAN CIMT & $\begin{array}{l}\text { RETINAL } \\
\text { ARTERY } \\
\text { CHANGES }\end{array}$ \\
\hline 0.9284 & 12 \\
\hline 0.6695 & 2 \\
\hline
\end{tabular}

\begin{tabular}{|l|l|l|l|}
\hline & RIGHT CIMT & LEFT CIMT & MEAN \\
\hline NAFLD & 0.9284 & 0.9284 & 0.9284 \\
\hline CONTROLS & 0.667 & 0.672 & 0.669 \\
\hline
\end{tabular}




\section{Association of mean carotid intima mediathickness and retinal artery:-}

In our study, it s clear that, increase in CIMT cause increase in retinal artery changes. The collected data were subjected to statistical analysis using Statistical package for Social sciences [SPSS version 19.0 (SPSS Inc., Chicago, IL, USA)]. Categorical data were presented as numbers and percentages, whereas continuous data were expressed as mean and standard deviation (SD) or median and inter quartile range. The continuous data was assessed for normality by Shapiro-wilk test. The pattern of distribution of each variable was found to be in non normal distribution thus non-parametric Mann-Whitney U test was indicated as appropriate test..

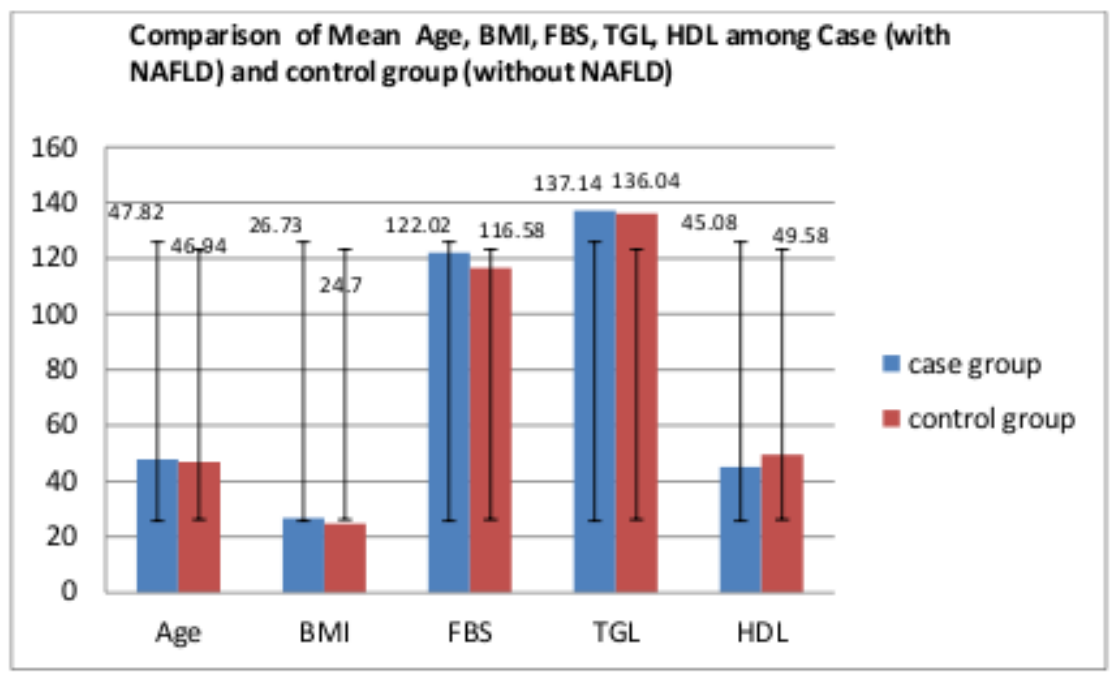

Relation OfNafld And Other Variables:-

1. There is strong association between carotid intima media thickness and NAFLD as p value is 0.001 .

2. There is strong association between HDL and NAFLD as pvalue is 0.001

3. There is strong association between BMI and NAFLD as pvalue is 0.008

4. There is strong association between RETINAL ARTERY CHANGES and NAFLD as p value is 0.004

5. There is strong association between SEX and NAFLD as pvalue is 0.001.(as female prevalence is more in NAFLD patients)

\begin{tabular}{|c|c|c|c|c|}
\hline \multicolumn{5}{|c|}{ Mann-Whitney Test } \\
\hline \multicolumn{2}{|c|}{ SUBJECTS } & $\mathrm{N}$ & MEAN RANK & SUM OF RANKS \\
\hline AGE & $\begin{array}{l}\text { CONTROLS } \\
\text { CASES } \\
\text { TOTAL }\end{array}$ & $\begin{array}{c}50 \\
50 \\
100\end{array}$ & $\begin{array}{l}49.38 \\
51.62\end{array}$ & $\begin{array}{l}2469.00 \\
2581.00\end{array}$ \\
\hline BMI & $\begin{array}{l}\text { CONTROLS } \\
\text { CASES } \\
\text { TOTAL }\end{array}$ & $\begin{array}{l}50 \\
50 \\
100\end{array}$ & $\begin{array}{l}42.87 \\
58.13\end{array}$ & $\begin{array}{l}2143.50 \\
2906.50\end{array}$ \\
\hline R-CIMT & $\begin{array}{l}\text { CONTROLS } \\
\text { CASES } \\
\text { TOTAL }\end{array}$ & $\begin{array}{c}50 \\
50 \\
100 \\
\end{array}$ & $\begin{array}{l}26.67 \\
74.33 \\
\end{array}$ & $\begin{array}{l}1333.50 \\
3716.50\end{array}$ \\
\hline L-CIMT & $\begin{array}{c}\text { CONTROLS } \\
\text { CASES } \\
\text { TOTAL }\end{array}$ & $\begin{array}{l}50 \\
50 \\
100\end{array}$ & $\begin{array}{l}26.87 \\
74.13\end{array}$ & $\begin{array}{l}1343.50 \\
3706.50\end{array}$ \\
\hline FBS & $\begin{array}{c}\text { CONTROLS } \\
\text { CASES } \\
\text { TOTAL }\end{array}$ & $\begin{array}{l}50 \\
50 \\
100 \\
\end{array}$ & $\begin{array}{l}45.43 \\
55.57\end{array}$ & $\begin{array}{l}2271.50 \\
2778.50\end{array}$ \\
\hline TGL & $\begin{array}{l}\text { CONTROLS } \\
\text { CASES } \\
\text { TOTAL }\end{array}$ & $\begin{array}{l}50 \\
50 \\
100\end{array}$ & $\begin{array}{l}51.80 \\
49.20\end{array}$ & $\begin{array}{l}2590.00 \\
2460.00\end{array}$ \\
\hline $\mathrm{HDL}$ & $\begin{array}{l}\text { CONTROLS } \\
\text { CASES } \\
\text { TOTAL }\end{array}$ & $\begin{array}{l}50 \\
50 \\
100\end{array}$ & $\begin{array}{l}61.15 \\
39.85\end{array}$ & $\begin{array}{l}3057.50 \\
1992.50\end{array}$ \\
\hline
\end{tabular}




\section{Test Statistics}

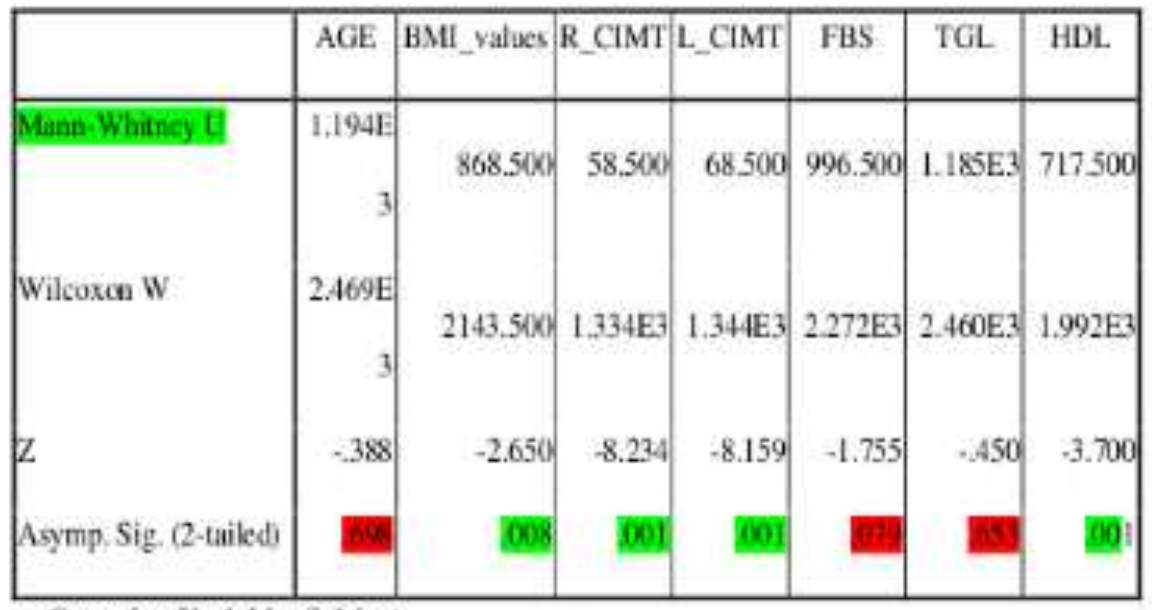

a. Grouping Variable: Subjects

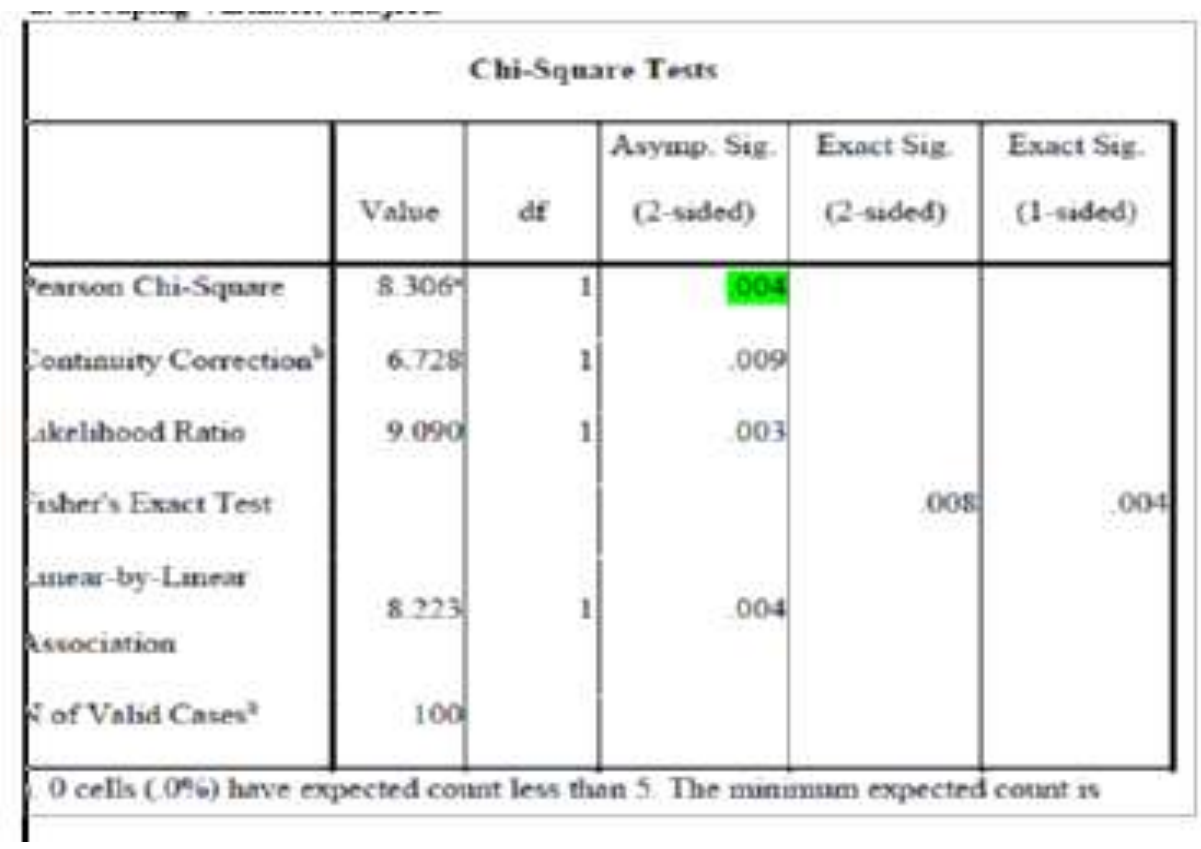

\section{Discussion:-}

In this study, CIMT and retinal artery changes in NAFLD patients was compared with control, which showed that mean CIMT and retinal artery changes were significantly much more than the control group. The results of our study are consistent with results of previous studies thatare considered CIMT was associated with an increase in NAFLD and suggested it as the marker of early diagnosis of generalized atherosclerosis (Chiang et al., 2010; Guleriaet al., 2013; Targher and Arcaro, 2007; Targheret al., 2008). This actually means that type II diabetic patients with NAFLD are at greater risk of premature atherosclerosis and Coronary Vessels Disease (CVD). As we stated before ultrasound screening method is a cheap and readily available. NAFLD estimates for up to one third of the total population and in the majority of patients with cardiovascular, metabolic and abdominal obesity, type II diabetes risk factors, can be seen. Our findings revealed NAFLD effect on CIMT was significant. In agreement with this finding, De Andrade et al. (2014) measured CIMT in a cross-sectional study on diabetes patients and showed that CIMT and CVD risk may be higher in those with a family history of type II diabetes. Besides, Nahandiet al. (2014) evaluated the effect of NAFLD on CIMT as a risk factor for atherosclerosis in patients with type II diabetes and reported that there is asignificant association between the presence of NAFLD and CIMT and its related atherosclerosis. 
Mohammadiet al. (2011) examined patients with confirmed NAFLD for determination of CIMT and presence of carotid atherosclerotic plaque and reported that NAFLD with type II diabetes can be associated with increased CIMT and increased risk of atherosclerosis. Moreover, Han et al. (2013) studied gender differences in the association between CIMT in healthy individuals and age-related increases in CIMT were correlated with a reduction in cardiac function only in women. Our findings showed a considerable association between NAFLD and increased CIMT, in which this association is not affected by the severity of fatty liver. And also considerable association between retinal artery chages and NAFLD also observedConsiderable association between NAFLD and obesity and HDL levels are also observed.

The internal carotid artery provides blood to the eye, therefore the pathology due to arteriosclerosis of these arteries may have a direct impact on retinal circulation and may coexist with retinal arteriosclerosis. So in our study, it is clear that, increase in carotid intima media thickness cause increased retinal artery changes. Data explaining CIMT relates to future cardiovascular events are very few.

Salonen and Salonen is the one of the few study available to date performed in a random sample $(\mathrm{n}=1257)$ of middle aged finnish men, reported that increase in 0.1mm of CIMT was associated with an $11 \%$ increase in the risk of cardiovascular complication. Hence all our NAFLD patients are treated with statins and other cardioprotective drugs, and also they are advised to follow up regularly once in every 6 months. They are advised to take treatment for NAFLD with insulinsensitizers. So, immediate ultrasound screening and treatment for the patients with NAFLD are recommended to prevent CVD complications such as atherosclerosis considering early stages of fatty liver disease.

\section{Conclusion:-}

In our study, we found that, there is strong association between, NAFLD and Carotid intima media thickness, Retinal artery changes. we also found significant association between NAFLD and body mass index and HDL levels. So all patients with NAFLD should be investigated with carotid Doppler, and they all should treated with statins and other cardioprotective drugs to prevent cardiovascular complication..This simple and non-invasive practice will help in early diagnosis of cardiovascular disease, especially in overt cases

\section{References}

1. Ables, G.P., 2012. Update on Pparyand nonalcoholic fatty liver disease. PPAR Res. 10.1155/2012/912351.

2. Aygun, C., O. Kocaman, T. Sahin, S. Uraz and A.T. Eminle ret al., 2008. Evaluation of metabolic syndrome frequency and carotid artery intima-media thickness as risk factors for atherosclerosis in patients with nonalcoholic fatty liver disease. Digestive Dis. Sci., 53: 1352-1357.

3) Babb, R.R., 2002. Nonalcoholic fatty liver disease. N. Engl. J. Med, 346: 1221- 1231.

4) Ballestri, S., A. Lonardo, S. Bonapace, C.D. Byme, P. Loria and G. Tapgher, 20-14 Risk of cardiovascular, cardiac and arrhythmic complic ations in patients with non-alcoholic fatty liver disease. World $\mathrm{J}$. Gastroenteiol., 20: 1724- 1745.

5) Int. J. Osteoporosis Metab. Disorders, 8 (2): 35-41, 2015 Bhatia, L.S., N.P. Cuuzen, P.C. Calder and C.D. Byme, 2012. Non-alcoholic fatty liver dise ase:

6) A new and important cardiovascular risk factor? Eur. Heart J., 33: 1190-1200. Bots, M.L. and D.E. Grobbee, 2002. Intima media thickness as a surrogate marker for generalized atherosclerosis. Cardiovasc. Drugs Ther., 16: 341-351. Brea, A and J. Puzo, 2013.Non-alcoholic fatty liver disease and cardiovascular risk.

7) Int. J. Cardiol, 167: 1109-1117 Caserta, C.A., GM. Pendino, A. Amante, C. Vacalebre and M.T. Finrilloet al, 2010.

8) Cardiovas cular risk factors, nonalcoholic fatty liver disease and carotid artery intima-media thickness in an adole scent population in Southern Italy. Am. J. Epidemiol, 171: 1195-1202. Chiang, C.H., C.C. Huang, W.L. Chan, J.W. Chen and H.B. Leu, 2010.

9) The severity of non-alcoholic fatty liver disease correlates with high sensitivity C-reactive protein value and is independently associated with increased cardiovascular risk in healthy population. Clin.Biochem. 43 : 1399-1404. Coll, B and C. Alonso-Villaverde, 2005.

10) Carotid intima-media thickness: As sessment of sub-clinical atherosc lerosis in HIV-infected patients. ADS, 19: 1936-1937. De Andrade, Jr. C.R., E.L. Silva, MF.B. da Matta, MB. Castier, M.L.G. Rosa, MB. Gomes, 2014. 